\title{
PERFIL DE LIBERAÇÃO IN VITRO DE DICLOFENACO DE SÓDIO INCORPORADO EM PARTÍCULAS DE SERICINA E ALGINATO
}

\author{
M. E. NAKASHIMA ${ }^{1}$, J. M. VIDART ${ }^{1}$, M. G. C. DA SILVA $^{1}$ e M. G. A. VIEIRA ${ }^{1}$ \\ ${ }^{1}$ Universidade Estadual de Campinas, Faculdade de Engenharia Química \\ E-mail para contato: melissagav@feq.unicamp.br
}

\begin{abstract}
RESUMO - Este trabalho teve como finalidade estudar a incorporação do fármaco diclofenaco de sódio em uma matriz formada pelos polímeros sericina (obtido do casulo do bicho-da-seda) e alginato (obtido comercialmente). Foram produzidas 3 formulações de partículas, as quais já haviam obtido porcentagens de incorporação altas em estudos anteriores, com diferentes proporções de sericina, alginato e fármaco. Essas partículas foram submetidas a ensaios de liberação em meio gástrico e entérico simulados, apresentando gastrorresistência das partículas e liberação controlada do fármaco devido, principalmente, à presença do alginato e da sericina, respectivamente. Ademais, foram aplicados diferentes modelos matemáticos à curva de liberação em meio tamponado $\mathrm{pH} 6,8$, sendo que o Modelo de Weibull foi o que apresentou melhores resultados $\left(\mathrm{R}^{2}{ }_{\text {aj }}\right.$ próximo de $1 \mathrm{e}$ menores valores de Critério de Informação de Akaike) em comparação com os demais ajustes.
\end{abstract}

\section{INTRODUÇÃO}

O casulo do bicho-da-seda é composto por duas principais proteínas: a fibroína e a sericina. A fibroína é utilizada nas indústrias de fiação de seda, enquanto a sericina é tratada como resíduo desse processo. Quando isolada, a sericina apresenta característica de gel; contudo, quando misturada com outros polímeros, obtém-se um material que pode ser aplicado em diversas áreas, como a de cosmético e de fármaco (Aramwit et al., 2012).

O alginato é um polissacarídeo derivado das algas marinhas, sendo caracterizado por sua gastrorresistência, o que o torna favorável para a incorporação de substâncias que são sensíveis a esse meio (SINHA et al., 2015). Desta forma, a formação de uma blenda entre a sericina e alginato torna-se interessante, uma vez que resulta em uma matriz adequada para a incorporação de fármacos.

O diclofenaco de sódio é um medicamento amplamente utilizado no tratamento de artrite reumatoide e osteoartrite. Por possuir uma meia-vida de 1-2 h, este fármaco requer múltiplas dosagens, causando efeitos adversos como gastrite e úlcera peptídica (Nayak e Pal, 2011). Assim, torna-se vantajosa a formulação de uma matriz, para a sua incorporação, que seja gastrorresistente.

O presente estudo teve como objetivo a preparação da blenda entre a sericina e o alginato, para a incorporação do fármaco diclofenaco de sódio. Avaliou-se os processos de 
liberação deste fármaco em meio gastrointestinal simulado, bem como o ajuste matemático que melhor representava a liberação deste fármaco em solução de tampão fosfato pH 6,8.

\section{MATERIAIS E MÉTODOS}

\subsection{Processo de Extração da Sericina}

Os casulos do bicho-da-seda foram obtidos da empresa de fiação de seda BRATAC. Eles foram abertos, limpos manualmente, cortados em pequenos pedaços e lavados. Após o processo de secagem dos casulos em estufa a $50{ }^{\circ} \mathrm{C}$, por cerca de $12 \mathrm{~h}$, os mesmos foram deixados em dessecadores de sílica-gel para resfriarem. A partir dos estudos de Tomadon Jr. (2011), adotou-se o método mais eficiente de extração, o qual ocorre em autoclave na pressão manométrica de $1 \mathrm{~kg}_{\mathrm{f}} \cdot \mathrm{cm}^{-2}$ e temperatura de $120{ }^{\circ} \mathrm{C}$ durante $40 \mathrm{~min}$. Utilizou-se uma proporção de $40 \mathrm{~g}$ de casulos secos para $1 \mathrm{~L}$ de água deionizada. A solução obtida foi armazenada em recipiente fechado a temperatura ambiente por $12 \mathrm{~h}$ e, posteriormente, foi disposta em congelador convencional durante $24 \mathrm{~h}$. Após esse período, a solução foi descongelada, filtrada em papel filtro com porosidade de $14 \mu \mathrm{m}$ e sua concentração foi ajustada para $2,5 \%(\mathrm{~m} / \mathrm{v})$.

\subsection{Formulação das Partículas}

Vidart et al. (2016) estudaram quatro formulações variando a proporção de sericina e alginato para a incorporação de diclofenaco de sódio. $\mathrm{O}$ presente trabalho procedeu-se à análise da liberação in vitro de três dessas formulações, apresentadas na Tabela 1, e que obtiveram melhores características morfológicas e eficiências de incorporação satisfatórias no estudo prévio.

Tabela 1 - Composição e eficiência de incorporação de cada formulação preparada

\begin{tabular}{|c|c|c|c|c|}
\hline Formulação & Sericina (g) & Alginato (g) & $\begin{array}{c}\text { Diclofenaco de } \\
\text { sódio (g) }\end{array}$ & $\begin{array}{c}\text { Eficiência de } \\
\text { incorporação (\%) }\end{array}$ \\
\hline \hline DS1 & 2,5 & 2,6 & 2,0 & 82,5 \\
\hline DS2 & 2,5 & 3,3 & 2,0 & 77,9 \\
\hline DS3 & - & 4 & 2,0 & 75,5 \\
\hline
\end{tabular}

\subsection{Liberação do Fármaco em Meios Simulados}

O teste de liberação in vitro do fármaco foi realizado conforme monografia do diclofenaco de sódio, apresentada pela Farmacopéia Brasileira. Sendo assim, para simular o meio gástrico, uma quantidade de partículas equivalente a $50 \mathrm{mg}$ de diclofenaco de sódio foi colocada em contato com $900 \mathrm{~mL}$ de ácido clorídrico $0,1 \mathrm{M}(\mathrm{pH} 1,2)$ durante $2 \mathrm{~h}$, em aparelho dissolutor de cestos, Logan modelo UDT-814-6, a $37 \pm 0,5^{\circ} \mathrm{C}$ e agitação de $50 \mathrm{rpm}$. Após esse período, uma alíquota de $5 \mathrm{~mL}$ foi coletada para determinar a quantidade de fármaco liberado, por meio da medição de absorbância em espectrofotômetro Shimadzu, modelo UV mini 1240, no comprimento de onde de $276 \mathrm{~nm}$. 
Após $2 \mathrm{~h}$ de ensaio em meio ácido, as partículas foram recuperadas dos cestos e colocadas em contato com $900 \mathrm{~mL}$ de tampão fosfato $\mathrm{pH}$ 6,8 (simulação do meio entérico) por $10 \mathrm{~h}$, em aparelho dissolutor de cestos nas mesmas condições em que o teste em meio ácido foi conduzido. Alíquotas de $5 \mathrm{~mL}$ foram coletadas, com reposição de meio de dissolução, em intervalos de tempo pré-determinados, para a determinação da concentração de fármaco liberado, por espectrofotometria UV-visível a $276 \mathrm{~nm}$.

\subsection{Cinética e Modelagem da Liberação do Fármaco}

Os modelos de ordem zero, primeira ordem, Weibull, Higuchi, Korsmeyer-Peppas e Hopfenberg para sistemas cilíndricos e esféricos foram aplicados aos dados da dissolução de diclofenaco de sódio usando o software Maple $\AA$, sendo que os ajustes foram analisados pelo coeficiente de determinação $\left(\mathrm{R}_{\mathrm{aj}}^{2}\right)$ e pelo critério de informação de Akaike (AIC). Os melhores ajustes possuem o coeficiente de determinação próximo de 1 e o menor valor de AIC.

\section{RESULTADOS}

A partir da Figura 1, percebe-se que as formulações DS1 e DS2 possuem uma maior esfericidade, quando comparadas com a formulação sem sericina DS3, que apresenta formato irregular. Tal fato indica a importância da presença da sericina na formulação para obter uma maior esfericidade das partículas.

Figura 1 - Partículas das três formulações produzidas.

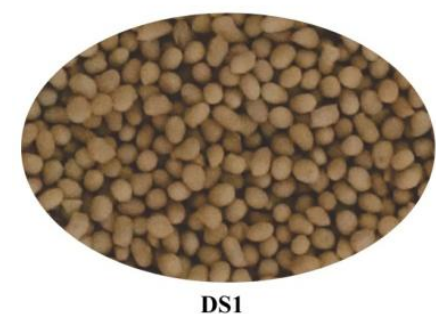

DS1

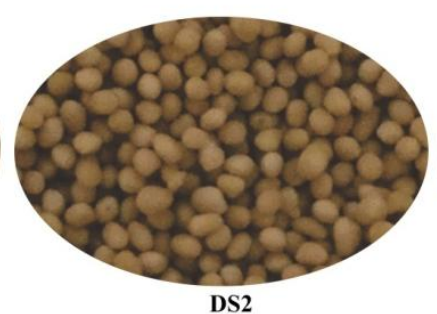

DS2

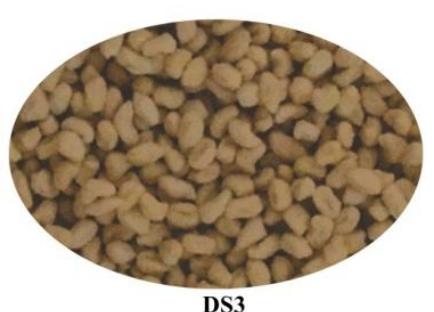

DS3

A Tabela 2 mostra os resultados obtidos no processo de dissolução em meio gástrico simulado. Os dados apresentados indicam que quanto maior a quantidade de alginato na formulação, menor é a quantidade de fármaco liberado nesse meio, ou seja, maior é a sua gastrorresistência. Ademais, segundo a Farmacopeia Brasileira, é permitido uma liberação de até $10 \%$ em meio ácido, indicando que os resultados obtidos são aceitáveis.

Tabela 2 - Porcentagem de liberação em meio ácido

\begin{tabular}{|c|c|}
\hline Formulação & $\begin{array}{c}\text { Liberação de } \\
\text { fármaco }(\%)\end{array}$ \\
\hline \hline DS1 & $2,63 \pm 0,66$ \\
\hline DS2 & $1,92 \pm 0,36$ \\
\hline DS3 & $1,49 \pm 0,26$ \\
\hline
\end{tabular}


A Figura 2 apresenta os perfis de dissolução das partículas em meio entérico simulado, sendo que as formulações DS1 e DS2 apresentaram uma total liberação entre 240-300 min, enquanto que o DS3 possui uma liberação mais acelerada, em $45 \mathrm{~min}$. Dessa forma, evidencia-se a importância da sericina na composição da partícula como um material que ajuda a retardar o processo de liberação do diclofenaco de sódio, tornando-o mais controlado e, consequentemente, minimizando os efeitos colaterais causados pelo fármaco.

Figura 2 - Perfil de liberação em meio entérico simulado.
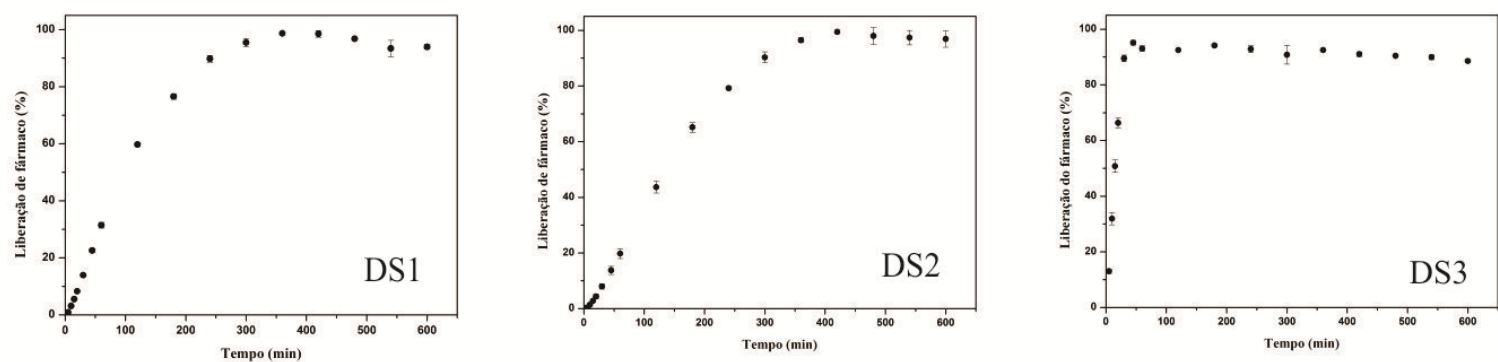

Na Tabela 3 constam os resultados obtidos, em que os modelos matemáticos de ordem zero, primeira ordem, Weibull, Higuchi, Korsmeyer-Peppas, Hopfenberg para sistemas cilíndricos $(n=2)$ e Hopfenberg para sistemas esféricos $(n=3)$ foram analisados. Conforme valores destacados, tem-se que o modelo de Weibull foi o que apresentou melhores ajustes para todas as formulações, uma vez que os valores de $\mathrm{R}_{\text {aj }}^{2}$ possuem os valores mais próximos de 1 e os valores de AIC são os menores em comparação com os demais. Ademais, obteve-se um valor do parâmetro de forma $b$ maior de 1 , caracterizando a curva como sigmoide, em forma de "S", com curvatura para cima seguida de um ponto de inflexão (Costa e Lobo, 2001).

Entre os resultados obtidos para o modelo de ordem zero, tem-se que a formulação DS3 obteve o pior ajuste, uma vez que sua hipótese é baseada na liberação de fármaco de maneira lenta e, como visto no perfil de dissolução obtido, esta formulação não teve uma liberação controlada como as demais. O modelo de Hopfenberg para sistema cilíndrico e esférico apresentou bons ajustes para todas as formulações, indicando que a liberação do fármaco é influenciada pelo processo de erosão, o qual é limitante desta ação. $\mathrm{O}$ modelo que apresentou o pior ajuste para todas as formulações foi o de Higuchi, o qual assume que o processo de difusão baseia-se na Lei de Fick, portanto, a liberação do diclofenaco de sódio incorporado à matriz de sericina e alginato não segue este princípio. Tal fato é reiterado pelos resultados obtidos para o Modelo de Korsmeyer-Peppas, visto que os valores de n (parâmetro de caracterização do mecanismo de liberação) estão entre 0,5 e 1,0, indicando que se trata de um processo de transferência de massa não-Fickiano (Costa, 2002). 
Tabela 3 - Modelagem matemática para a liberação de diclofenaco de sódio em solução tampão fosfato $\mathrm{pH}=6,8$

\begin{tabular}{|c|c|c|c|c|c|c|c|c|}
\hline \multirow[b]{2}{*}{ Formulações } & \multirow[b]{2}{*}{ Parâmetros } & \multicolumn{7}{|c|}{ Modelos } \\
\hline & & $\begin{array}{c}\text { Ordem } \\
\text { zero }\end{array}$ & $\begin{array}{c}\text { Primeira } \\
\text { ordem }\end{array}$ & Weibull & Higuchi & $\begin{array}{c}\text { Korsmeyer- } \\
\text { Peppas }\end{array}$ & $\begin{array}{c}\text { Hopfenberg; } \\
\mathbf{n}=\mathbf{2}\end{array}$ & $\begin{array}{l}\text { Hopfenberg; } \\
\qquad \begin{array}{l}\mathbf{n}=\mathbf{3}\end{array}\end{array}$ \\
\hline \multirow{6}{*}{ DS1 } & $\mathrm{k}$ & 0,3706 & 0,0074 & - & 5,1394 & 1,3055 & 0,0028 & 0,0021 \\
\hline & $\mathrm{n}$ & - & - & - & - & 0,7671 & - & - \\
\hline & $\mathrm{a}$ & - & - & 542,55 & - & - & - & - \\
\hline & $\mathrm{b}$ & - & - & 1,2935 & - & - & - & - \\
\hline & $\mathrm{R}_{\mathrm{aj}}^{2}$ & 0,9101 & 0,9509 & 0,9991 & 0,7226 & 0,9496 & 0,9924 & 0,9873 \\
\hline & AIC & 74,17 & 63,22 & 21,88 & 81,17 & 66,12 & 44,71 & 49,53 \\
\hline \multirow{6}{*}{ DS2 } & $\mathrm{k}$ & 0,3002 & 0,0055 & - & 4,5118 & 0,5997 & 0,0021 & 0,0016 \\
\hline & $\mathrm{n}$ & - & - & - & - & 0,8763 & - & - \\
\hline & $\mathrm{a}$ & - & - & 2234,54 & - & - & - & - \\
\hline & $\mathrm{b}$ & - & - & 1,4941 & - & - & - & - \\
\hline & $\mathrm{R}_{\mathrm{aj}}^{2}$ & 0,9520 & 0,9027 & 0,9993 & 0,6421 & 0,9574 & 0,9759 & 0,9585 \\
\hline & AIC & 73,43 & 78,33 & 22,44 & 93,44 & 71,77 & 63,16 & 69,09 \\
\hline \multirow{6}{*}{ DS3 } & $\mathrm{k}$ & 2,5551 & 0,0507 & - & 13,92 & 7,3167 & 0,0199 & 0,0144 \\
\hline & $\mathrm{n}$ & - & - & - & - & 0,6969 & - & - \\
\hline & $\mathrm{a}$ & - & - & 91,72 & - & - & - & - \\
\hline & $\mathrm{b}$ & - & - & 1,5378 & - & - & - & - \\
\hline & $\mathrm{R}_{\mathrm{aj}}^{2}$ & 0,7797 & 0,8175 & 0,9939 & 0,6193 & 0,8144 & 0,9537 & 0,9271 \\
\hline & AIC & 43,07 & 36,72 & 19,40 & 41,24 & 39,37 & 30,02 & 32,11 \\
\hline
\end{tabular}

\section{CONCLUSÕES}

A partir dos resultados apresentados, evidenciou-se a importância da sericina para a esfericidade das partículas. Ademais, o teste de dissolução em meios simulados indicou a gastrorresistência das partículas, devido, principalmente, à presença do alginato na composição, bem como a importância da sericina para uma liberação mais controlada do 
diclofenaco de sódio em meio tamponado. Os resultados dos ajustes dos modelos matemáticos apresentou melhores resultados para o modelo de Weibull, devido aos valores de $\mathrm{R}^{2}{ }_{\text {aj }}$ e AIC serem os mais próximos do ideal.

\section{Agradecimentos}

Os autores agradecem à BRATAC pelo fornecimento dos casulos, Geolab ${ }^{\circledR}$ Indústria Farmacêutica S/A pelo fornecimento do diclofenaco de sódio, CNPq (Proc. 470615/2013-3 e 300986/2013-0), CAPES e FAPESP (Proc. 2015/13505-9) pelo suporte financeiro.

\section{REFERÊNCIAS}

ARAMWIT, P.; SIRITIENTONG, T.; SRICHANA, T. Potential applications of silk sericin, a natural protein from textile industry by-products. Waste management and Research, v. 30, p. 217-224, 2012.

COSTA, P.; LOBO, J. M. S. Modeling and comparison of dissolution profiles. European Journal of Pharmaceuticals Science, v. 13, p. 123-133, 2001.

COSTA, P. J. C. Avaliação in vitro da lioequivalência de formulações farmacêuticas. Revista Brasileira de Ciências Farmacêuticas, v. 38, n. 2, abr./jun., 2001.

NAYAK, A. K.; PAL, D. Development of pH-sensitive tamarind seed polysaccharidealginate composite beads for controlled diclofenac sodium delivery using response surface methodology. International Journal of Biological Macromolecules, v.49, p.784793, 2011.

SINHA, P.; UBAIDULLA, U.; HASNAIN, M. S.; NAIAK, A. K.; RAMA, B. Alginate-okra gum blend beads of diclofenac sodium from aqueous template using $\mathrm{ZnSO} 4$ as a crosslinker. International Journal of Biological Macromolecules, v. 79, p. 555-563, 2015.

TOMADON JR, J. Obtenção da proteína sericina, com alta massa molecular, a partir de casulos Bombyx mori. Paraná: Universidade Estadual de Maringá, Dissertação (Mestrado), p. 172, 2011.

VIDART, J. M. M.; NAKASHIMA, M.; SILVA, T. L.; ROSA, P. C. P.; GIMENES, M. L.; VIEIRA, M. G. A.; SILVA, M. G. C. Sericin and Alginate Blend as Matrix for Incorporation of Diclofenac Sodium. Chemical Engineering Transactions, v. 51, p. $343-$ 348, 2016. 